\title{
Gain Adaptation for Continuous Sliding Mode Control
}

\author{
Hancheol Cho \\ Department of Aerospace and Mechanical Engineering \\ University of Liège \\ Liège, Belgium \\ hancheol.cho@gmail.com; hancheol.cho@ulg.ac.be
}

\begin{abstract}
In this paper a novel adaptive sliding mode controller design is presented for robust control of nonlinear uncertain systems. A continuous control law to compensate for the uncertainties is first developed that is completely free from chattering. Focusing on the relation between the tried gain value and the resultant sliding variable, a new method for estimating the uncertainty bounds is then derived, leading to an adaptive law for gain-tuning by which the error eventually lies within a user-specified region in a finite time. Unlike other existing approaches, the new adaptive rule only requires the magnitude of the control input in the previous time step, which greatly eases the application of the proposed algorithm to real-world systems. An inverted pendulum system is simulated to demonstrate the accuracy and effectiveness of the proposed control strategy.
\end{abstract}

Keywords-sliding mode control; gain adaptation; Lyapunov stability

\section{INTRODUCTION}

Control of nonlinear systems in the presence of uncertain parameters and disturbances is a main topic in the modern control theory. Sliding mode control (SMC) has attracted numerous researchers due to its high robustness, insensitivity to matched uncertainties, and computational efficiency. However, conventional SMC usually suffers from 'chattering', which results in high-frequency oscillations in the control input and/or in the state. The most common way to circumvent this problem is to use a boundary layer approach or a quasi-sliding mode approach [1,2]. However, these approaches sometimes do not completely remove chattering as noted in [3] and are not adequate for the stability proof of the controlled system. A more elegant method that alleviate chattering is to introduce high-order sliding mode control (HOSMC) [4-6]. However, the discontinuous structure is still used in the controller design of this approach, thereby causing a little chattering though it is considerably disappeared.

One more drawback of HOSMC is the requirement of the $a$ priori knowledge of the bounds on the uncertainties. In practice, it is pretty difficult to exactly estimate these bounds, and hence, the concept of adaptive sliding mode control (ASMC) was proposed. In ASMC, the gain, representing the bounds on the uncertainties, is updated at each time step so that it is as small as possible to reduce chattering but simultaneously sufficient enough to counteract the effects of

Hancheol Cho is a Marie Curie COFUND Postdoctoral Fellow at the University of Liège in Belgium. the uncertainties. Huang et al. [7] proposed an adaptive law in which the gain increases until the sliding mode is achieved. However, in this approach the gain never decreases, sometimes yielding severe chattering. Also, only the asymptotic stability of the controlled system is proven so it is not guaranteed that the error converges to zero in a finite time. In [8] a novel approach in which the gain is dynamically updated so that it increases if the sliding mode is lost and decreases if the system is in the sliding mode. Also, a finite-time convergence of the controlled system is proven. This approach is extended to multi-input multi-output systems in [9]. In [10] a continuous control law with adaptive gain is newly proposed. Although the controller does not place the sliding variable exactly onto the sliding manifold, one can always force the sliding variable to be bounded within a user-specified small domain. Also, simple gain estimation and adaptation methods are proposed in which the gain is updated according to whether the controlled system is in the sliding mode or not. However, in this approach the gain always increases so if the initial estimate for the gain is larger than the real unknown value, the updated gain is overestimated. This gain overestimation can lead to excessive chattering especially if the saturation of the control input is considered.

The main goal of this paper is to propose a new continuous control law with a simple gain adaptation method. An adaptive gain-tuning method that exploits the relation between the applied gain and the resultant sliding variable is developed. The approached adopted in this paper is similar to, but differs from, the one suggested in [10], in that the gain can now either increase or decrease while the adaptive gain is always greater than the upper bound on the uncertainties. The gain adaptation requires only the magnitude of the control input in the previous time step, which significantly eases its application to a real-life system. The robustness and the effectiveness of the proposed adaptive control scheme is verified by solving an inverted pendulum problem with uncertain parameters and disturbances.

\section{Problem Formulation}

In this paper the following nonlinear uncertain system is considered: 


$$
\begin{gathered}
\dot{\boldsymbol{x}}(t)=\boldsymbol{f}(t, \boldsymbol{x}(t))+\boldsymbol{g}(t, \boldsymbol{x}(t)) u(t), \\
y(t)=h(\boldsymbol{x}(t)),
\end{gathered}
$$

where $\boldsymbol{x}(t) \in \mathbf{R}^{n}$ is the state vector, $u(t) \in \mathbf{R}$ is the control input, $y(t) \in \mathbf{R}$ is the system output, and $h(\boldsymbol{x})$ is the output function. It is assumed that functions $\boldsymbol{f}(t, \boldsymbol{x})$ and $\boldsymbol{g}(t, \boldsymbol{x}) \neq 0$ are smooth, uncertain, and bounded for $\boldsymbol{x}$. Denoting the error vector $e(t)$ as

$$
e(t)=y(t)-y_{d}(t)
$$

where $y_{d}(t)$ is the desired output, then the sliding variable $s(t)$ is defined as follows:

$$
s(t):=e^{(n-1)}(t)+\lambda_{n-1} e^{(n-2)}(t)+\cdots+\lambda_{2} \dot{e}(t)+\lambda_{1} e(t),
$$

where the constant coefficients $\lambda_{n-1}, \cdots, \lambda_{2}, \lambda_{1}$ comprise a Hurwitz polynomial. In (3), the superscript $(n-1)$ denotes the $(n-1)$ th time derivative. Assume that $y^{(n)}(t)$ can be represented by

$$
y^{(n)}(t)=\xi(t, \boldsymbol{x})+b(t, \boldsymbol{x}) u(t),
$$

where $\xi(t, \boldsymbol{x})$ and $b(t, \boldsymbol{x}) \neq 0$ are smooth and unknown. It is noted that since the relative degree of the system (1) is equal to the system's order, the system (1) does not have internal dynamics. From here on for brevity, the arguments of the various quantities will be suppressed unless required for clarity. The time derivative of the sliding variable (3) leads to

$$
\begin{aligned}
\dot{s} & =e^{(n)}+\lambda_{n-1} e^{(n-1)}+\cdots+\lambda_{2} \ddot{e}+\lambda_{1} \dot{e} \\
& =\left(y^{(n)}-y_{d}^{(n)}\right)+\lambda_{n-1} e^{(n-1)}+\lambda_{2} \ddot{e}+\lambda_{1} \dot{e} \\
& =\left(\xi+b u-y_{d}^{(n)}\right)+\lambda_{n-1} e^{(n-1)}+\lambda_{2} \ddot{e}+\lambda_{1} \dot{e} \\
& =z+b u,
\end{aligned}
$$

where $z(t, x):=\left(\xi-y_{d}^{(n)}\right)+\lambda_{n-1} e^{(n-1)}+\cdots+\lambda_{2} \ddot{e}+\lambda_{1} \dot{e}$ is defined. In this paper, the functions $z(t, \boldsymbol{x})$ and $b(t, \boldsymbol{x})$ are supposed to be bounded by

$$
|z(t, \boldsymbol{x})|<\Gamma_{r}, 0<b_{r}<b(t, \boldsymbol{x})<B_{r} .
$$

It is noted that the positive constants $\Gamma_{r}, b_{r}$, and $B_{r}$ exist, but are not known.

\section{CONTROller Design}

This section proposes a chattering-free sliding mode controller with adaptive gain. The main feature of the approach concerns the fact that it does not require a priori knowledge of the uncertainty bounds $\left(\Gamma_{r}\right.$ and $\left.b_{r}\right)$. First, the continuous control law in the following form is considered:

$$
u=-\frac{\Gamma_{r}}{\varepsilon b_{r}} s
$$

where $\varepsilon$ is a small positive. Then, the following theorem holds.

Theorem 1 Given the nonlinear uncertain system (1) controlled by (7), the state trajectories of (1) are forced to move from initial conditions to the region $|s(t)| \leq \varepsilon$ in a finite time and remain in the region thereafter.

Proof. Let us define a Lyapunov function $L(t)$ taking the form:

$$
L=\frac{1}{2} s^{2}
$$

Differentiating (8) with respect to time yields

$$
L=s \dot{s},
$$

and substituting (9) into (8), one gets

$$
\begin{aligned}
\dot{L} & =s z-\frac{\Gamma_{r} b}{b_{r} \varepsilon} s^{2}<\Gamma_{r}|s|-\frac{\Gamma_{r} b}{b_{r} \varepsilon} s^{2} \\
& =\Gamma_{r}|s|\left(1-\frac{b}{b_{r} \varepsilon}|s|\right)=-\sqrt{2} \Gamma_{r}\left(\frac{b}{b_{r} \varepsilon}|s|-1\right) \frac{|s|}{\sqrt{2}} \\
& =-\alpha \cdot V^{1 / 2},
\end{aligned}
$$

where $\alpha=\sqrt{2} \Gamma_{r}\left(\frac{b}{b_{r} \varepsilon}|s|-1\right)$ is a positive parameter in the region where $|s(t)|>\varepsilon$ holds. Hence, the state trajectories of the nonlinear system (1) controlled by (7) converge to the region $|s(t)| \leq \varepsilon$ in a finite time and remain in the region thereafter, which completes the proof.

Corollary 1 Let us consider an $n$ th-order system described by (1) and suppose that the sliding variable $s$ given by (3) is bounded by $|s| \leq \varepsilon$. Then for a second-order system $(n=2)$, the actual error $e(t)$ is eventually bounded by 


$$
|e(t)| \leq \frac{\varepsilon}{\lambda_{1}}
$$

as time progresses. For a third-order system $(n=3)$, the actual error $e(t)$ is eventually bounded by

$$
\begin{aligned}
& |e(t)| \leq \frac{\varepsilon}{\lambda_{1}}, \quad \text { when } \lambda_{2}^{2} \geq 2 \lambda_{1}, \\
& |e(t)| \leq \frac{2 \varepsilon}{\lambda_{2} \sqrt{4 \lambda_{1}-\lambda_{2}^{2}}}, \text { when } \lambda_{2}^{2}<2 \lambda_{1} .
\end{aligned}
$$

Proof. For a second-order system, the sliding variable is given by (3) as follows:

$$
s=\dot{e}+\lambda_{1} e,
$$

where $\lambda_{1}$ is a positive constant. Then, by the assumption $|s| \leq \varepsilon$, one has

$$
-\varepsilon \leq \dot{e}+\lambda_{1} e \leq \varepsilon
$$

and let us first consider the following condition:

$$
\dot{e}+\lambda_{1} e \leq \varepsilon .
$$

Using the Grönwall's inequality [11], (15) leads to

$$
e \leq\left(e_{0}-\frac{\varepsilon}{\lambda_{1}}\right) \exp \left[-\lambda_{1}\left(t-t_{0}\right)\right]+\frac{\varepsilon}{\lambda_{1}}
$$

where $e_{0}:=e\left(t_{0}\right)$ is defined. Next, consider the following condition:

$$
-\varepsilon \leq \dot{e}+\lambda_{1} e,
$$

then, following the same procedure one can obtain the following result.

$$
e \geq\left(e_{0}+\frac{\varepsilon}{\lambda_{1}}\right) \exp \left[-\lambda_{1}\left(t-t_{0}\right)\right]-\frac{\varepsilon}{\lambda_{1}} .
$$

Combining (16) and (18), the error is bounded by

$$
\left(e_{0}+\frac{\varepsilon}{\lambda_{1}}\right) \exp \left[-\lambda_{1}\left(t-t_{0}\right)\right]-\frac{\varepsilon}{\lambda_{1}} \leq e \leq\left(e_{0}-\frac{\varepsilon}{\lambda_{1}}\right) \exp \left[-\lambda_{1}\left(t-t_{0}\right)\right]+\frac{\varepsilon}{\lambda_{1}} .
$$

Since $\lambda_{1}$ is positive, the exponential term decays rapidly as time progresses so that

$$
-\frac{\varepsilon}{\lambda_{1}} \leq e \leq \frac{\varepsilon}{\lambda_{1}} .
$$

Next, let us consider a third-order system in which the sliding variable is described by

$$
s=\ddot{e}+\lambda_{2} \dot{e}+\lambda_{1} e,
$$

where $\lambda_{1}$ and $\lambda_{2}$ are positive constants. In this case, the Grönwall's inequality does not apply, and hence, another approach should be attempted. First, by the assumption, one has

$$
\left|\ddot{e}+\lambda_{2} \dot{e}+\lambda_{1} e\right| \leq \varepsilon .
$$

(22) can be thought of as a mass-springer-damper system driven by an external force whose maximum magnitude is $\varepsilon$. According to the vibration theory, the maximum magnitude of $|e|$ is obtained when the system is at resonance. Resonance occurs only when $\lambda_{2}^{2}<2 \lambda_{1}$ holds and the corresponding maximum resonant magnitude of $|e|$ is obtained as [12]

$$
\max (|e|)=\frac{2 \varepsilon}{\lambda_{2} \sqrt{4 \lambda_{1}-\lambda_{2}^{2}}} .
$$

If $\lambda_{2}^{2} \geq 2 \lambda_{1}$ holds, resonance does not occur and in this case the maximum magnitude of $|e|$ is independent of $\lambda_{2}$ and given by

$$
\max (|e|)=\frac{\varepsilon}{\lambda_{1}}
$$

and this completes the proof.

Up to now, it has been shown that if the control law (7) with the bounds $\Gamma_{r}$ and $b_{r}$ is applied to the nonlinear uncertain system (1), the sliding variable $s(t)$ is eventually bounded in a finite time within a region where $|s| \leq \varepsilon$ holds. Since (7) consists of the continuous function $s(t)$, it is inherently chattering free. Although one cannot exactly place the sliding variable onto the sliding manifold $s(t)=0$, it is always possible to arbitrarily choose a small number $\varepsilon$ so that the error is confined in a user-specified domain.

In the use of the control law (7), however, the knowledge of the bounds $\Gamma_{r}$ and $b_{r}$ is prerequisite. In general, an accurate determination of these bounds is very difficult, so it is highly 
desirable to develop an adaptive rule by which the uncertain bounds are automatically tuned so that finite-time convergence described in Theorem 1 is guaranteed without a priori information about the bounds.

Now, let us define a gain $K_{r}$ as the ratio of $\Gamma_{r}$ to $b_{r}$, i.e., $K_{r}=\Gamma_{r} / b_{r}$, and then (7) is rewritten as

$$
u=-\frac{K_{r}}{\varepsilon} s
$$

Suppose that $\Gamma_{r}$ and $b_{r}$ are exactly determined, then the sliding variable will be eventually bounded by $|s| \leq \varepsilon$ as shown by Theorem 1. Provided that the gain is overestimated and $2 K_{r}$ is applied instead of $K_{r}$, then the sliding variable would be bounded by $|s| \leq \varepsilon / 2$ because increasing the gain twice is equivalent to decreasing $\varepsilon$ by half, that is,

$$
u=-\frac{2 K_{r}}{\varepsilon} s=-\frac{K_{r}}{(\varepsilon / 2)} s
$$

More generally, if a gain guesstimate $\hat{K}$ is tried, then the control law (7) becomes

$$
u=-\frac{\hat{K}}{\varepsilon} s=-\frac{K_{r}}{\left(\varepsilon K_{r} / \hat{K}\right)} s
$$

and one would observe that the sliding variable is bounded by $|s| \leq \hat{\varepsilon}$, where $\hat{\varepsilon}=\varepsilon K_{r} / \hat{K}$. In brief, in order to find the real unknown gain $K_{r}$ one can try a rough guesstimate $\hat{K}$ and observe the upper bound $\hat{\varepsilon}$ on $|s|$ so that $|s| \leq \hat{\varepsilon}$. Then, the real gain $K_{r}$ can be found by

$$
K_{r}=\frac{\hat{\varepsilon}}{\varepsilon} \hat{K}
$$

where $\varepsilon$ is the desired upper bound on $|s|$. (27) reveals a very interesting result on the maximum magnitude of the control input $u(t)$. From (27), one has

$$
\max (|u|)=\frac{\hat{K}}{\varepsilon} \max (|s|)=\frac{\hat{K}}{\varepsilon} \cdot \frac{\varepsilon K_{r}}{\hat{K}}=K_{r}=\text { const. }
$$

The maximum magnitude of the control input, $\max (|u|)$, is equal to the unknown real gain $K_{r}=\Gamma_{r} / b_{r}$, which is always constant and independent of the choice of the gain. This striking fact and the new method (28) for the estimation of the gain leads to an adaptive rule as follows.

Consider the control law given by

$$
u(t)=-\frac{K(t)}{\varepsilon} s(t)
$$

and the gain $K(t)$ is dynamically updated in real time by the following adaptive law:

$$
K^{(k+1)}=\left|u^{(k)}\right|+K_{m}
$$

where the superscript $(k)$ denotes a quantity at the $k$ th scan time and $K_{m}$ is a positive constant. The adaptive rule (31) is based on the fact that $|s|$ should be always kept less than $\varepsilon$. From (30), one has

$$
\frac{|u|}{K}=\frac{|s|}{\varepsilon}
$$

In order to have $|s| \leq \varepsilon$, the following condition should be satisfied from (32):

$$
K \geq|u|
$$

which leads to the adaptive rule (31). Let us first prove the following lemma:

Lemma 1. Given the nonlinear uncertain system (1) controlled by (30) and (31), then the gain $K(t)$ has an upper bound, i.e., there exists a positive constant $K^{*}$ so that $K(t) \leq K^{*}$ for all $t>0$.

Proof. First, it is noted from (27) that if the gain $K=K_{r}+K_{m}$ is applied, the sliding variable is bounded by

$$
|s| \leq \varepsilon \cdot \frac{K_{r}}{K_{r}+K_{m}}=\varepsilon\left(1-\frac{K_{m}}{K}\right), \text { when } K=K_{r}+K_{m}
$$

Next, the derivative of the gain $K$ is approximately represented by (31):

$$
\dot{K} \simeq \frac{K\left(\frac{|s|}{\varepsilon}-1\right)+K_{m}}{\Delta t},
$$

where $\Delta t$ is the step size, and (35) yields 


$$
\begin{aligned}
& \dot{K}>0 \text {, when }|s|>\varepsilon\left(1-\frac{K_{m}}{K}\right), \\
& \dot{K}<0 \text {, when }|s|<\varepsilon\left(1-\frac{K_{m}}{K}\right) .
\end{aligned}
$$

Also, from (5), one has

$$
\begin{aligned}
& \dot{s}>0 \text {, when } K<\frac{\varepsilon}{b} \frac{|z|}{|s|}, \\
& \dot{s}<0 \text {, when } K>\frac{\varepsilon}{b} \frac{|z|}{|s|} .
\end{aligned}
$$

Suppose that, at $t=t_{0},\left|s\left(t_{0}\right)\right|>\varepsilon\left(1-K_{m} / K\left(t_{0}\right)\right)$ and $\dot{s}\left(t_{0}\right)>0$ (i.e., $|s|$ is increasing). It means that the gain $K\left(t_{0}\right)$ is less than $K_{r}+K_{m}$ as indicated in (34). Then, $K$ starts to increase by (36). For $t>t_{0}, K$ can either increase or decrease according to the conditions (36) and (37), however, as the "worst" case, let us assume that $K$ and $|s|$ are continuously increasing. If

$$
K\left(t_{1}\right)>\frac{\varepsilon}{b\left(t_{1}\right)} \frac{\left|z\left(t_{1}\right)\right|}{\left|s\left(t_{1}\right)\right|}
$$

holds at $t=t_{1},|s|$ starts to decrease from (37). Here, $K$ can again either increase or decrease according to (36) and (37). If $K$ is continuously increasing as the worst case, it will eventually become greater than $K_{r}+K_{m}$. Then, Theorem 1 and (34) state that there exists a finite time $t_{2}$ so that

$$
\left|s\left(t_{2}\right)\right| \leq \varepsilon\left(1-\frac{K_{m}}{K\left(t_{2}\right)}\right)
$$

holds and $K$ starts to decrease according to (36). $K$ keeps decreasing until $K<K_{r}+K_{m}$ holds because if $K$ is greater than $K_{r}+K_{m}$, then $|s|$ is always less than $\varepsilon\left(1-K_{m} / K\right)$ and the second condition of (36) is satisfied. After that, $K$ and $|s|$ can keep decreasing or again increase whether the conditions (36) or (37) are met or not. Again, if the worst case is assumed so that at $t=t_{3},\left|s\left(t_{3}\right)\right|>\varepsilon\left(1-K_{m} / K\left(t_{3}\right)\right)$ and $K$ starts to increase, then the analysis starts from the very beginning. In brief, the gain $K(t)$ cannot increase unlimitedly because when it is greater than $K_{r}+K_{m}$, the sliding variable becomes less than $\varepsilon\left(1-K_{m} / K\right)$ in a finite time as in (39) and $K(t)$ starts to decrease and keeps decreasing until it becomes smaller than $K_{r}+K_{m}$. In addition, from (29) and (31) one can readily deduce that the maximum $K(t)$ is the sum of the maximum magnitude of $u(t)$ and $K_{m}$, which is constant and finite. Hence, Lemma 1 is proven.

Theorem 2. Given nonlinear uncertain system (1) with the sliding variable (3) controlled by (30) and (31), then there exists a finite time $t_{F}>0$ so that the condition $|s(t)| \leq \varepsilon$ is satisfied for all $t \geq t_{F}$.

Proof. Let us define the following Lyapunov candidate function:

$$
V=\frac{1}{2} s^{2}+\frac{1}{2 \gamma}\left(K-K^{*}\right)^{2}
$$

where $\gamma$ is a positive constant. It should be noted that the condition $K \leq K^{*}$ by Lemma 1 .

The time derivative of the Lyapunov function (40) yields

$$
\begin{aligned}
\dot{V} & =s \dot{s}+\frac{1}{\gamma}\left(K-K^{*}\right) \dot{K} \\
& =s(z+b u)+\frac{1}{\gamma}\left(K-K^{*}\right) \dot{K} \\
& =s\left(z-\frac{b K}{\varepsilon} s\right)+\frac{1}{\gamma}\left(K-K^{*}\right) \dot{K} \\
& <|s|\left(\Gamma_{r}-\frac{b K}{\varepsilon}|s|\right)+\frac{1}{\gamma}\left(K-K^{*}\right) \dot{K},
\end{aligned}
$$

where $|z|<\Gamma_{r}$ is used.

First, the case when $|s|>\varepsilon$ is considered. Then, (41) satisfies

$$
\begin{aligned}
\dot{V} & <|s|\left(\Gamma_{r}-b K\right)+\frac{1}{\gamma}\left(K-K^{*}\right) \dot{K} \\
& =|s|\left(\Gamma_{r}-b K\right)+\frac{1}{\gamma}\left(K-K^{*}\right) \dot{K}+|s| b K^{*}-|s| b K^{*} \\
& =-\beta_{s}|s|-\left|K-K^{*}\right|\left(-b|s|+\frac{1}{\gamma} \dot{K}\right),
\end{aligned}
$$

where $\quad \beta_{s}=b_{m} K^{*}\left(b / b_{m}-1\right)>0$ is defined and $K-K^{*}=-\left|K-K^{*}\right|$ is used because $K \leq K^{*}$. Now, a parameter $\beta_{K}>0$ is introduced in (42) as

$$
\begin{aligned}
\dot{V} & <-\beta_{s}|s|-\left|K-K^{*}\right|\left(-b|s|+\frac{1}{\gamma} \dot{K}\right)+\beta_{K}\left|K-K^{*}\right|-\beta_{K}\left|K-K^{*}\right| \\
& =-\beta_{s}|s|-\beta_{K}\left|K-K^{*}\right|-\kappa,
\end{aligned}
$$

where $\kappa=\left|K-K^{*}\right|\left(-b|s|+\dot{K} / \gamma-\beta_{K}\right)$ is defined. Finally, (43) leads to 


$$
\begin{aligned}
\dot{V} & <-\beta_{s} \cdot \sqrt{2} \frac{|s|}{\sqrt{2}}-\beta_{K} \cdot \sqrt{2 \gamma} \frac{\left|K-K^{*}\right|}{\sqrt{2 \gamma}}-\kappa \\
& \leq-\min \left\{\beta_{s} \sqrt{2}, \beta_{K} \sqrt{2 \gamma}\right\}\left(\frac{|s|}{\sqrt{2}}+\frac{\left|K-K^{*}\right|}{\sqrt{2 \gamma}}\right)-\kappa \\
& \leq-\beta \cdot V^{1 / 2}-\kappa,
\end{aligned}
$$

where $\beta=\sqrt{2} \min \left\{\beta_{s}, \beta_{K} \sqrt{\gamma}\right\}>0$.

It is noted that it is always possible to make $\kappa>0$ by a proper selection of $\gamma$ that is not included in a design parameter of control. The condition $\kappa>0$ yields

$$
-b|s|+\frac{1}{\gamma} \dot{K}-\beta_{K}>0 \Rightarrow \gamma<\frac{\dot{K}}{b|s|+\beta_{K}} .
$$

With (35), (45) becomes

$$
\gamma<\frac{K\left(\frac{|s|}{\varepsilon}-1\right)+K_{m}}{\Delta t\left(b|s|+\beta_{K}\right)} .
$$

Now, with the condition $|s|>\varepsilon, \gamma$ can be selected so that it is smaller than the right hand side of (46), or

$$
\gamma<\frac{K_{m}}{\Delta t_{\max }\left(b_{M} \varepsilon+\beta_{K}\right)}
$$

where $\Delta t$ is the maximum step size and $B_{r}$ is the upper bound for $b$ as noted in (6).

Finally, from (27), one has $\dot{V}<-\beta \cdot V^{1 / 2}-\kappa<-\beta \cdot V^{1 / 2}$, and hence, finite time convergence to the region $|s| \leq \varepsilon$ is guaranteed from the time when $|s|$ starts to exceed $\varepsilon$. The convergence time is calculated as

$$
t_{F} \leq \frac{2 V^{1 / 2}(0)}{\beta} .
$$

Next, let us consider the case when $|s| \leq \varepsilon$ holds. In this case, $\kappa$ may not be positive so that $\dot{V}$ is sign-indefinite and $|s|$ may exceed $\varepsilon$. However, as soon as it becomes larger than $\varepsilon, \dot{V}<-\beta \cdot V^{1 / 2}$ holds and $|s|$ will be again bounded by $|s| \leq \varepsilon$ in a finite time, as shown earlier.

In brief, the adaptive rule (31) guarantees that the sliding variable will be bounded within the region $|s| \leq \varepsilon$ in a finite time $t_{F}$ from any initial condition and will remain in the region thereafter, which completes the proof.

\section{NUMERICAL EXAMPLE}

To demonstrate the effectiveness and the robustness of the adaptive control scheme proposed in this paper, an inverted pendulum problem with uncertain parameters and disturbances is introduced. The simulations in this example are carried out in the Matlab/Simulink environment, using the ode4 RungeKutta integrator.

Let us consider the following inverted pendulum system:

$$
\ddot{x}+c(t) \dot{x}+a \cos (x)=b u+d(t),
$$

where $x$ and $t$ are normalized displacement and time, respectively. The (unknown) parameters of the actual system are $a=2.3, c(t)=1+0.3 \sin (t), b=0.9$, and the disturbance is $d(t)=3 \sin (0.25 t)$. Suppose that the output $y$ is $y=x$ and the desired trajectory is $y_{d}=\sin (0.5 t)$. Since (49) is a second-order system, the sliding variable is given by

$$
s(t)=\dot{e}(t)+\lambda_{1} e(t)
$$

where $\lambda_{1}=2$ is assumed. Also, the control law (30) with $\varepsilon=0.01$ and the adaptive rule (31) with $K_{m}=0.5$ are used for the simulation so that the resultant error is bounded by $|e| \leq \varepsilon / \lambda_{1}=0.005$ by Corollary 1 . The initial conditions are $x(0)=0$ and $\dot{x}(0)=0.5$ so that $e(0)=0$ and $\dot{e}(0)=0$ are assumed.

The result is shown in Fig. 1. In the very initial phase, $|s|$ exceeds $\varepsilon$, but it becomes bounded by $|s| \leq \varepsilon$ in a very short time and remains within the bounded region thereafter. Also, the error is always less than $\varepsilon / \lambda_{1}$ as noted in Corollary 1 .

In Fig. 2, the control input $u(t)$ and the adaptive gain $K(t)$ are depicted, being compared with the uncertainty $|z(t)| / b$, where

$$
z(t)=-c(t) \dot{x}-a \cos (x)+d(t)-\ddot{y}_{d}(t)+\lambda_{1}\left[\dot{x}-\dot{y}_{d}(t)\right]
$$

is obtained from (5). It is obvious that the control input does not suffer from chattering and the gain $K(t)$ is always greater than the uncertainty $|z(t)| / b$, which clearly shows that the proposed adaptive algorithm successfully updates the gain without knowledge of the bounds on the uncertainties. Furthermore, the only parameter needed for the adaptation is $K_{m}$ and it follows that the controller design is greatly 
simplified while the prominent robustness and the high accuracy are achieved.

In order to see the effect of the parameter $K_{m}$, its value is now reduced to 0.001 . The sliding variable $s(t)$ and the error $e(t)$ are plotted in Fig. 3. Compared with Fig. 1, they are exactly bounded by $|s| \leq \varepsilon$ and $|e| \leq \varepsilon / \lambda_{1}$, respectively. However, in the very initial phase the overshoot is larger than the one in Fig. 1 and the convergence time of $|s|$ into the region $|s| \leq \varepsilon$ takes longer than in Fig. 1 because the initial gain $K(0)=K_{m}=0.001$ is much smaller. Figure 4 displays the control input and the adaptive gain that are compared with the uncertainty $|z(t)| / b$. Since $K_{m}$ is very small, it is observed that the gain follows the uncertainty with little difference, resulting in the exact boundedness of $|s|$ on $\varepsilon$ as depicted in Fig. 3. To sum up, $K_{m}$ should be not selected as a very small number so as to guarantee a short convergence time and a small error.

\section{CONCLUSiOnS}

A novel adaptive sliding mode controller is proposed for a class of nonlinear systems with bounded uncertainties whose bounds are not known. Focusing on the relation between the applied gain and the resultant bound magnitude of the sliding variable, a simple adaptive law is derived. Since the gaintuning is implemented by just the sum of the magnitude of the control input in the previous time step and a positive constant, the suggested algorithm can be readily applied to real-world systems. The effectiveness of the proposed control scheme is verified by solving the inverted pendulum problem.
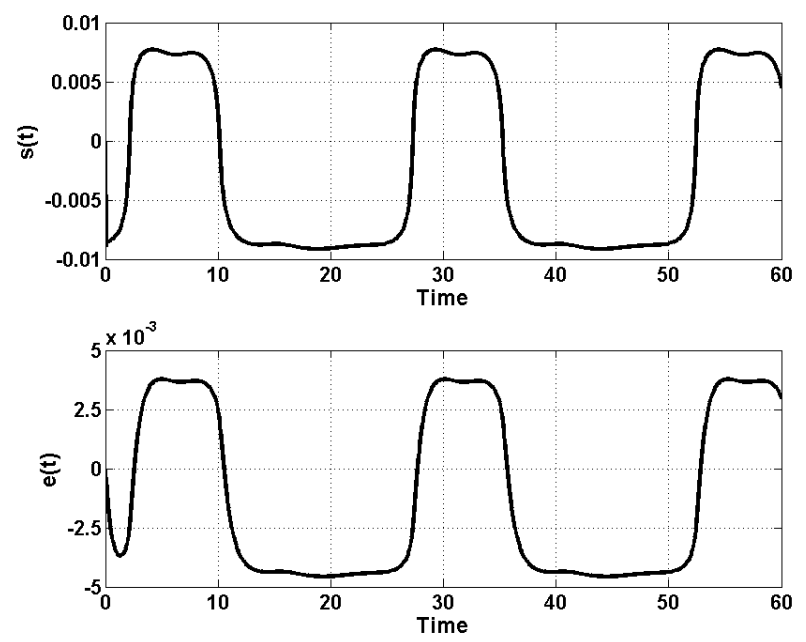

Fig. 1 Sliding variable $s(t)$ and error $e(t)$ when $K_{m}=0.5$
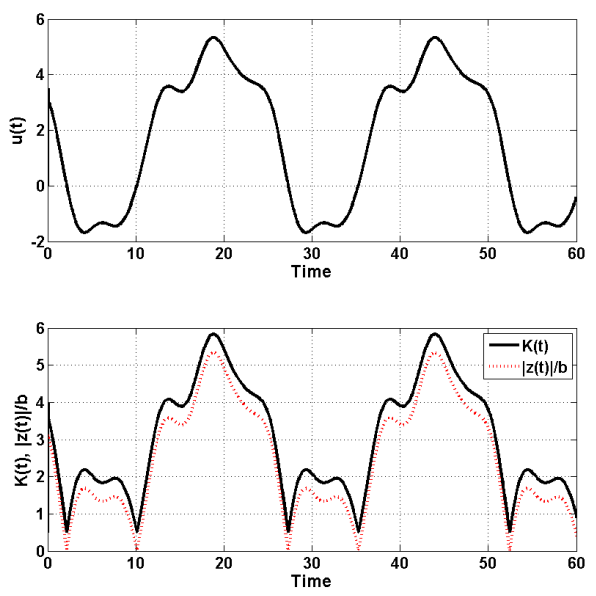

Fig. 2 Control input $u(t)$, adaptive gain $K(t)$, and uncertainty $|z(t)| / b$ when $K_{m}=0.5$
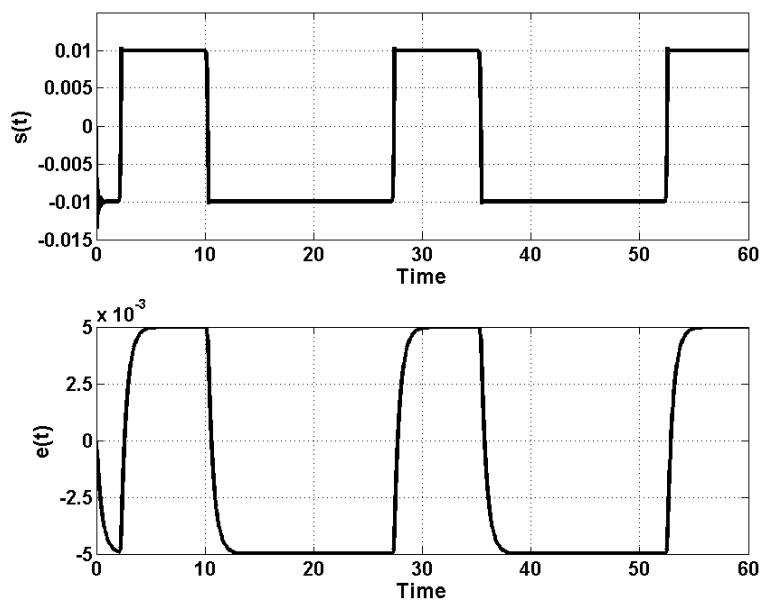

Fig. 3 Sliding variable $s(t)$ and error $e(t)$ when $K_{m}=0.001$
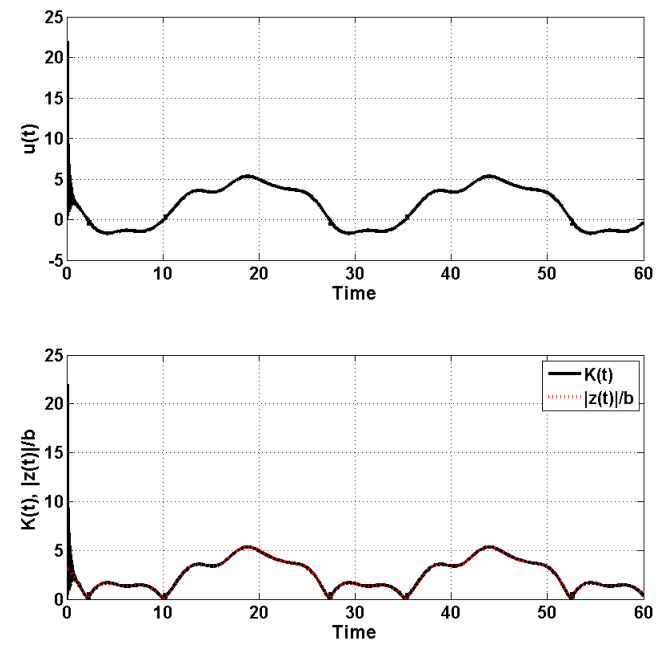

Fig. 4 Control input $u(t)$, adaptive gain $K(t)$, and uncertainty $|z(t)| / b$ when $K_{m}=0.001$ 


\section{REFERENCES}

[1] J. J. Slotine and S. S. Sastry, "Tracking control of nonlinear systems using sliding surfaces with application to robot manipulator," Int. J. Control, vol. 38, pp. 465-492, 1983.

[2] Y. Shtessel, C. Edwards, L. Fridman, and A. Levant, "Sliding mode control and observation," Springer, New York, 2014.

[3] M. Li, F. Wang, and F. Gao, "PID-based sliding mode controller for nonlinear processes," Ind. Eng. Chem., vol. 40, pp. 2660-2667, 2001.

[4] S. Laghrouche, F. Plestan, and A. Glumineau, "Higher order sliding mode control based on integral sliding surface," Automatica, vol. 43, pp. 531-537, 2007.

[5] A. Estrada and L. M. Fridman, "Integral HOSM semiglobal controller for finite-time exact compensation of unmatched perturbations," IEEE Trans. Automat. Contr., vol. 55, pp. 2645-2649, 2010.

[6] A. Levant, "Sliding order and sliding accuracy in sliding mode control," Int. J. Control, vol. 58, pp. 1247-1263, 1993.
[7] Y.-J. Huang, T.-C. Kuo, S.-H. Chang, "Adaptive sliding-mode control for nonlinear systems with uncertain parameters," IEEE Trans. Syst. Man Cy. B, vol. 38, pp. 534-539, 2008.

[8] F. Plestan, Y. Shtessel, V. Brégeault, and A. Poznyak, "New methodologies for adaptive sliding mode control," Int. J. Control, vol. 83, pp. 1907-1919, 2010.

[9] F. Plestan, Y. Shtessel, V. Brégeault, and A. Poznyak, "Sliding mode control with gain adaptation - Application to an electropneumatic actuator," Control Eng. Pract., vol. 21, pp. 679-688, 2013.

[10] H. Cho and G. Kerschen, "Simple adaptive methodology for chatteringfree sliding mode control," Proc. 14 ${ }^{\text {th }}$ International Workshop on Variable Structure Systems (VSS 2016), June 2016.

[11] T.H. Grönwall, "Note on the derivatives with respect to a parameter of the solutions of a system of differential equations," Ann. of Math., vol. 20, pp. 292-296, 1919.

[12] S.S. Rao, "Mechanical vibrations," 5th Ed. Prentice Hall, New Jersey, 2011 